\title{
Study on canine hip dysplasia and its diagnosis by imaging and molecular genetics techniques
}

\author{
Felix Daniel LUCACI ${ }^{1}$, Radu LĂCĂTUȘ ${ }^{1}$, Robert Cristian PURDOIU ${ }^{1}$ and \\ Dana Liana PUSTA ${ }^{1}$
}

\author{
${ }^{1}$ Faculty of Veterinary Medicine, University of Agricultural Sciences and Veterinary Medicine Cluj-Napoca, Mănăştur \\ St. 3-5, 400372 Cluj-Napoca, Romania. \\ *Corresponding author: Felix Daniel LUCACI e-mail: felix.lucaci@usamvcluj.ro
}

REVIEW

\begin{abstract}
The present paper is a bibliographical research on canine hip dysplasia using imaging and molecular genetics techniques. Ever since the first description in 1935 made by Schnelle, canine hip dysplasia has remained one of the most diagnosed orthopedic conditions present in the dog breeds. The gold standard method of diagnosis of hip dysplasia is the radiological examination in hip-extended position. While the radiological examination focuses mainly on the individual for the diagnosis of this condition, methods of diagnosis of an entire population are sought, and these methods are represented by molecular genetics techniques. Naming the etiology of canine hip dysplasia and finding out the latest methods of genetically and radiologically diagnosis of canine hip dysplasia and the best alternatives of treatment for this disease. Canine hip dysplasia continues to be a major problem for owners, breeders and veterinarians. Currently, there are five standardized systems worldwide that deal with the grading of canine hip dysplasia. In addition to digital radiology, CT and ultrasonographic examination are feasible diagnosis methods. Even if the standard method of diagnosis remains the radiological examination in the present, the near future provides to be of the molecular genetic techniques.
\end{abstract}

Keywords: canine hip dysplasia; CT; digital radiology; molecular genetics techniques; ultrasonography.

Received: 15 September 2020

Accepted: 20 April 2021

Published: 14 May 2021

DOI:

10.15835/buasvmcn-vm:2020.0031

\section{INTRODUCTION}

The present paper is a bibliographical research on canine hip dysplasia using imaging and molecular genetics techniques. We will make references to the etiology of the disease, the methods of diagnosis and treatment as well as certain aspects regarding the genetics of the disease.

Since it was first described by Schnelle in 1935, canine hip dysplasia has remained one of the most diagnosed orthopedic conditions present in the canine breed (King M., 2017).

Canine hip dysplasia is characterized by joint instability, subluxation, or even dislocation of the femoral head, which will result in a deformation of the joint with the early development of coxarthrosis (Mortellaro et al, 2008). The gold standard method of diagnosis is a radiological examination with the hip joint in forced extension (Schachner, and Mandi, 2015). Radiological screening of canine hip dysplasia had provided moderate success in decreasing the prevalence of this condition (Merca Roxana et al., 2020).

2021 Authors. The papers published in this journal are licensed under the Creative Commons Attribution-NonCommercialNoDerivatives 4.0 International License

\section{THE ETIOLOGY OF CANINE HIP DYSPLASIA}

\section{Genetic factors}

The literature presents two genes and a protein that could be directly related to canine hip dysplasia. 
The CHST3 gene is the one that encodes the synthesis of the sulfotransferase enzyme. In dysplastic dogs, a mutation in exon 3 of this gene causes the substitution of glycine with arginine, thus causing deficiencies in the formation of articular cartilage (Peterson C., 2017).

The FBN2 is a gene that encodes the synthesis of fibrillin II. Fibrillin II is located on chromosome 11 in dogs and has an important role in the elastic tissue of the extracellular matrix present in the joints, especially in the tendons. In dysplastic patients, it occurs a deletion of this gene from the chromosome causing changes in the connective tissue around the joint (Todhunter J. R., 2018).

Fibronectin I is a glycoprotein with a role in the extracellular matrix of cartilage. An increased amount of this glycoprotein has been found in dysplastic subjects (Peterson C., 2017).

\section{ENVIRONMENTAL FACTORS}

\section{Nutrition factors}

They are among the most important environmental factors in terms of the development of canine hip dysplasia. Studies have shown that an adequate nutritional intake, correct during the growing period, can reduce the risk of developing canine hip dysplasia (Kealy et al 1997).

Puppies do not have a protective mechanism against exogenous calcium intake, so calcium or vitamin D supplements decrease osteoclastic activity and normal ossification will be delayed, which may cause hip dysplasia in individuals with a genetic predisposition (King M, 2017).

\section{Exercise}

Krontveit, 2011, made an investigation on four large dog breeds (Labrador retriever, Irish wolfhounds, Newfoundlands, and Leonbergers) in Norway and found out that dogs that descend and climb stairs from birth to 3 months of age are more prone for developing hip dysplasia because during this period of life the musculoskeletal system is most vulnerable to the action of mechanical stress and the development of orthopedic disorders.

Another study, contradictory to the first one, also realized by Krontveit et al, 2012, shows that off-leash activity performed on soft ground, as early as possible in the life of the puppy increases muscle mass and strength in the coxo-femoral joint. However, the authors point out that any activity on the stairs should be avoided and physical effort should be moderate in duration and intensity.

\section{Body type}

In general, small breeds of dogs have a lower incidence of hip dysplasia compared to large and giant breeds. Heavy breeds, those with round conformation, poorly developed muscle mass, and those with early physical maturity are most susceptible to hip dysplasia, while breeds with well-defined muscles, with right limbs, are less prone because a strong muscle mass with a correct posture is considered to inhibit the transformation of passive joint laxity into functional laxity when the dog is in motion (Krontveit, 2012).

\section{Hormones}

Exogenous estrogen or relaxin administered to the puppies or to the pregnant bitches can accelerate the development of hip dysplasia by increasing joint laxity. Pregnant bitches with hip dysplasia may have a modified metabolism, and therefore puppies may be exposed to increased amounts of relaxin before birth. Colostrum contains estrogens and relaxin, and these can induce joint laxity in puppies with a predisposition to hip dysplasia. However, there is no clear evidence to confirm that relaxin, estrogen, or other hormones can play a decisive role in a biologically healthy field. (Krontveit, 2012, King M., 2017).

\section{Rate of growth}

In German Shepherd breed dogs, it was concluded that specimens that at 60 days of age were overweight will show in adulthood signs of hip dysplasia, compared to those with normal weight at 60 days of age. Excessive feeding can maximize the expression of canine hip dysplasia, but only in individuals with a genetic predisposition (Krontveit, 2012). Weight reduction for dogs was accepted as a preventive action or as a delay in clinical signs (Tobias, and Johnston, 2012).

\section{Diagnosis of canine hip dysplasia}

The diagnosis of the canine hip dysplasia is initially based on the anamnesis obtained by the veterinarian from the patient owner, then he will perform a functional examination following the patient at rest and while he is walking, after that the clinical examination is continued with the inspection, palpation and orthopedic examination. After the clinical examination, the veterinarian will continue with the paraclinical examination (Papuc, and Lăcătuş, 2013). 


\section{Radiological examination}

The radiological examination remains the gold standard method in the diagnosis of canine hip dysplasia. The first visible radiological sign is a radiopaque line from the base of the femoral neck to the trochanteric fossa, which is the result of new bone formations along the insertion of the joint capsule and is known as Morgan's line (Figure1.) (Thrall, 2018).

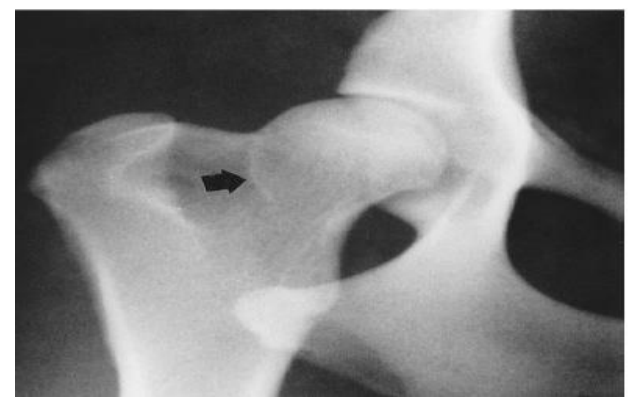

Figure 1. Morgan's line (black arrow)

Source: Thrall D.E., 2018

Worldwide there are five screening approaches for canine hip dysplasia: Federation Cynologique Internationale, British Veterinary Association/Kennel Club, the Orthopedic Foundation for Animals, Pennsylvania Hip Improvement Program, and Dorsolateral Subluxation Approach (Schachner and Mandi, 2015).

In Romania, as well as in countries that use either the British system or that of the international canine federation, for the diagnosis of canine hip dysplasia, among other measurements, the Norberg angle is also used as presented in Figure 2 $\mathrm{m}$ (Papuc et al, 2013).

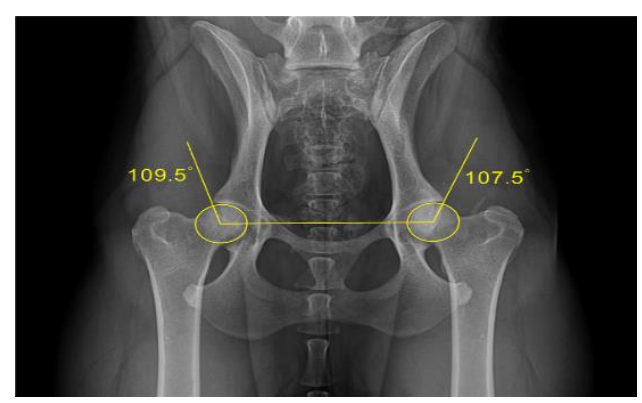

Figure 2. Norberg's angle

Source: www.superveterinary.com

\section{Federation Cynologique Internationale (FCI)}

Currently, the screening system for canine hip dysplasia at FCI is used in most countries in Europe, Russia, South America, South Africa, and Asia (Figure 3). The scoring system combines the subjective standard hip-extended radiographic evaluation with the Norberg's angle measurement.

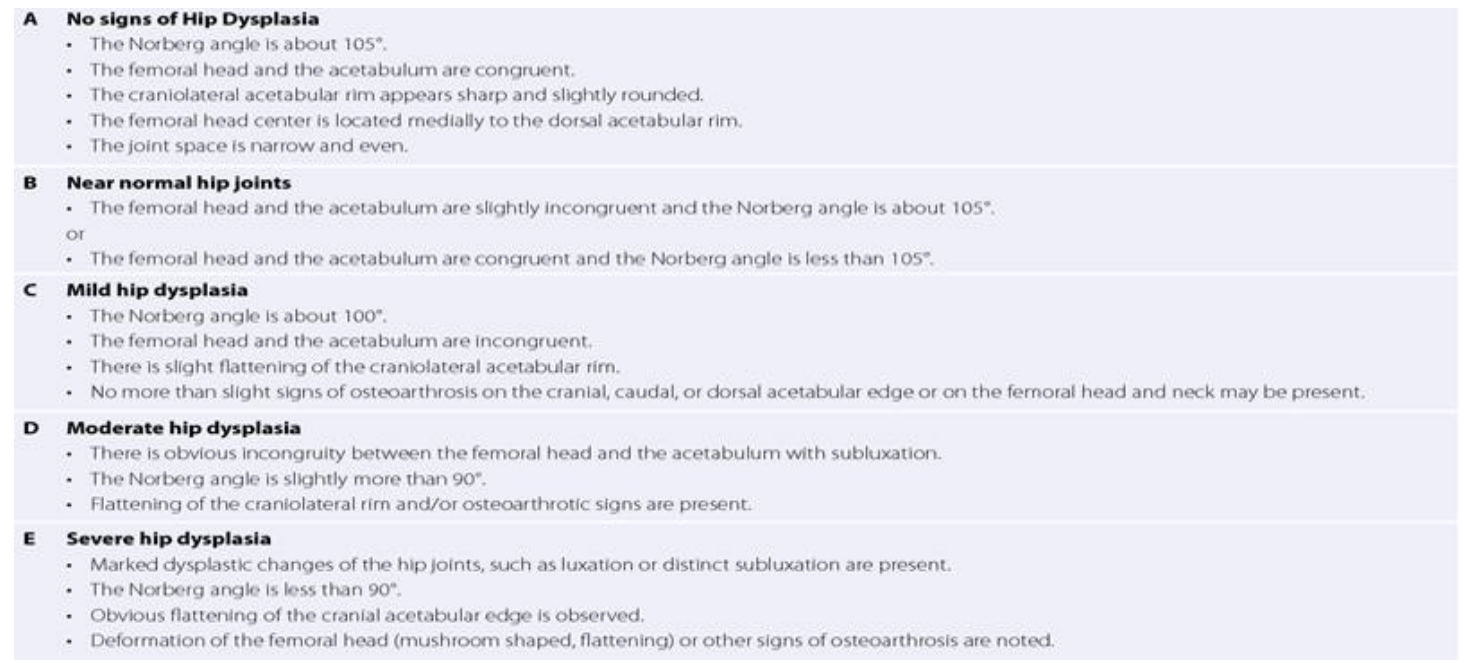

Figure 3. FCI scheme for grading CHD

Source : Kyriazis A. et al, 2016 
Norberg's angle is used in the ventrodorsally incidence for the pelvis and is a measurement of the relationship between the center of the femoral head and the margin of the acetabular cavity. Patients with a Norberg angle $\geq 105^{\circ}$ are considered non-dysplastic, while those with a Norberg angle below $105^{\circ}$ are diagnosed with varying degrees of dysplasia, depending on the congruence between the femoral head and the acetabular cavity, the presence or absence of osteoarthritis, and of thickening the acetabular cavity (Schachner, and Mandi, 2015; Papuc, and Lăcătuș, 2013). There are five grades, from A$\mathrm{E}$, where A-B is for non-dysplastic patients and $\mathrm{E}$ is a patient with severe hip-dysplasia. The minimum age for scoring is from 12-18 months of age (Schachner, and Mandi, 2015).

\section{British Veterinary Association/Kennel Club (BVA/KC)}

Since 1965, for the screening of canine hip dysplasia in the British system, dogs have been marked with ,pass” or „failed” in Great Britain, Ireland, and Australia. Radiographs are performed with the hips in forced extension, in patients at least one-year-old, and a score is obtained using nine criteria, and the score varies between 0-6. The left and right hips are measured separately, after which the sum is made, the interval being between 0-106 (53 for each hip) (Verhoeven Et al, 2011; Schachner, and Mandi, 2015).

\section{The Orthopedic Foundation for Animals (OFA)}

OFA is the organization that has been screening for canine hip dysplasia since 1966, in the United States and Canada. Dogs over 2 years of age are examined radiologically in the standard position with their hips in forced extension. Patients are classified according to the degree of dysplasia as follow: excellent, good, correct, borderline, mild dysplasia, moderate dysplasia, and severe dysplasia. Signs of incongruence and degenerative joint disease and hip joint laxity are considered scoring criteria (Figure 4). The scoring system does not use the Norberg angle measurement. Patients with the score excellent, good and correct are considered nondysplastic while dogs with mild, moderate, and severe are considered dysplastic patients (Schachner, and Mandi, 2015; Verhoeven Et al, 2011).

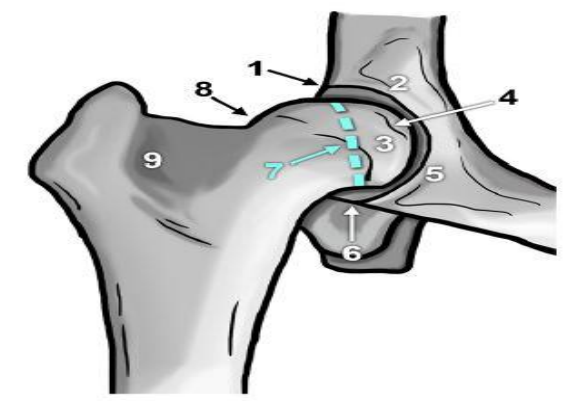

(a)

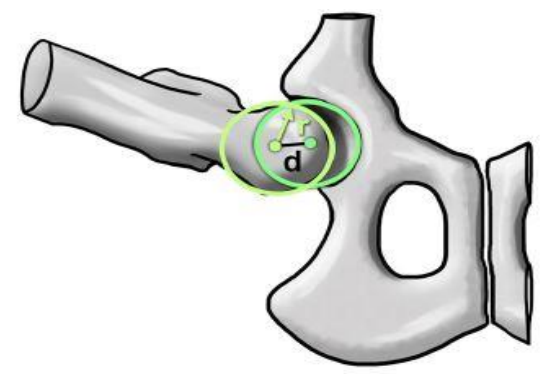

(b)

Figure 4. Hip joint anatomical characteristics and distraction index: (a): 1- craniolateral acetabular rim, 2-cranial acetabular margin, 3-femoral head, 4-fovea capitis, 5-acetabular notch, 6-caudal acetabular margin, 7-dorsal acetabular margin, 8-junction of femoral head and neck, and 9-trochanteric fossa (Source: Schachner et al, 2015); (b): the distance between femoral head and acetabulum (Source: Schachner et al, 2015).

\section{Pennsylvania Hip Improvement Program (PenHIPP)}

The PennHIP method is based on passive joint laxity, with the goal of detecting passive joint laxity as quickly as possible, at a minimum of 16 weeks of age. The patient will be in a recumbency position, and the femurs are held in a 10 $15^{\circ}$ extension, thus the proximal aspect of the femurs is compressed into the acetabulum obtaining the compression index. With a specially made distractor placed between the patient's pelvic limbs, the femoral bones will undergo an abduction thus allowing a lateral movement of the femoral head from the acetabulum. On the radiograph obtained in this position is calculated the distraction index (DI), which represents the distance between the center of the femoral head and the center of the acetabulum (Fig.5). Distraction index values are between 0-1, where 0 being total congruence between the center of the femoral head and the center of the acetabulum, and 1 meaning complete dislocation. Dogs with a score between 0-3 DI are unlikely to develop hip joint degeneration, while those with values above 0.7 DI, will certainly develop hip joint pathologies (Soo M. Et al, 2015; Verhoeven G. Et al, 2011).

\section{Dorsolateral Subluxation Approach (DLS)}

In the DLS system, the position of the dog is described as a weight-bearing position, thus DLS method obtained a more functional hip laxity than the PennHip method. The patient will be in a sternal position, on a special support that allows the 
knee joint to flex, so it will not be a bone overlap during the x-ray (Figure 6a). Both femoral bones are perpendicular to the radiological table, thus supporting body weight and putting additional stress on the coxo-femoral joint. On the dorsoventral radiograph, the DLS percent is obtained by calculating the percentage of femoral head that remains medial to the most lateral aspect of the cranial acetabular rim (Figure b). Patients with a DLS score above 55\% will be unlikely to develop canine hip dysplasia during their lifetime, those with a DLS score below $45 \%$ have chances for developing the disease. The DLS score is used more often in genetic studies (Saunders and Broeckx 2017; Verhoeven et al, 2011).

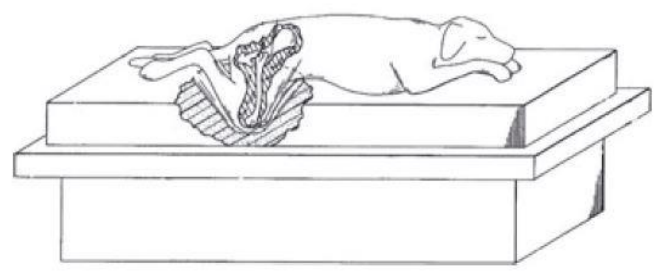

(a)

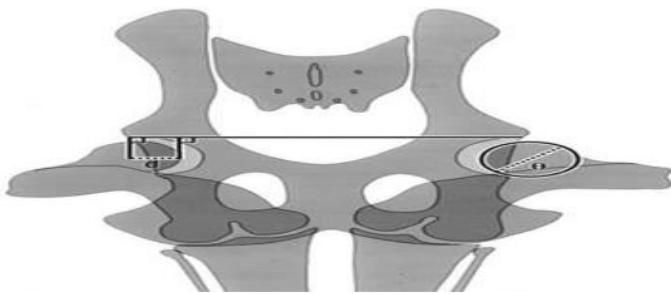

(b)

Figure 6. A. The Dorsolateral Subluxation position; B. The DLS score is calculated as a percentage $\mathrm{d} / 0 \mathrm{x} 100$, where $\mathrm{d}$ - distance between a line dropped from the cranial acetabular lateral margin and a parallel line, tangential with the most medial surface of femoral head; and 0- diameter of the best fitting circle of the femoral head (Source: Verhoeven et al., 2011).

\section{Computed tomography}

CT scan has become very popular in recent years in the diagnosis of canine hip dysplasia. CT images in two dimensions can measure: percentage of coverage of the femoral head, acetabular index and certain angles: acetabular, ventral and dorsal anteversion angle, horizontal acetabular sector, center edge angle (CEA) and on CT images in three dimensions can be measured: the volume of the head and neck of the femur, the rays of the head and of the femoral neck, and the angle of the femoral neck. The measurement of the center edge angle (CEA) and distraction index (DI) combined with the measure of the dorsal acetabular sector angle and Norberg angle at 16 and 32 weeks of age can have a predictive value on osteoarthritis in mature dogs (Butler, and Gambino, 2017; Schachner E.R. et al, 2015). Due to the high price for a single examination, a CT scan is not used as a routine method for diagnosing canine hip dysplasia.

\section{Ultrasound}

Ultrasonography is not a part of the current methodology for diagnosing this condition. The results are often contradictory, especially in puppies under 8 weeks. Ultrasonographic evaluation is based on a subjective evaluation of acetabular bone morphology and measurement of two angles $\alpha$ and $\beta$. At the ultrasound examination, there are three markers to be followed for being sure for the accuracy of the position: the caudal edge of the ilium in the acetabular fossa, the bone edge of the acetabular bone with the iliac silhouette, and the cartilage triangle 'labrum acetabulare' (Schachner and Mandi, 2015; Shipov et al., 2019).

\section{MRI}

Magnetic resonance, as an imaging modality, is not currently used for diagnosing canine hip dysplasia. Magnetic resonance can be used for the evaluation of ligamentous structures, soft tissues, joint capsules, and the proximal femoral physis. MRI technique is a non-invasive method that can be helpful for assessing the diseases of articular cartilage. With dGEMRIC (delayed gadolinium-enhanced MRI of cartilage) in conjunction with T1-weighted spin-echo an early stage of osteoarthritis can be revealed. In the T2 imaging modality, the sensitivity of tissue hydration and water content can be assessed, so an increase of the T2-weighted relaxation times is equivalent to cartilage damage. There are some other factors, such as the cost, time, the expertise required, and the need for general anesthesia for an increased length of time, which determines both owners and veterinarians to choose another imaging method for diagnosing canine hip dysplasia (Butler, and Gambino, 2017).

\section{Genetic tests}

Largely the current methods of diagnosis and treatment focus on the individual, while genetic testing seeks to solve the problem of an entire population. Current genetic studies are aimed at identifying genes responsible for canine hip dysplasia, genetic mutations, and quantitative trait loci (regions of chromosomes containing DNA for a specific trait) (Schachner, and Mandi, 2015). 
Pfahler and Distl, 2012, identified in their study three single nucleotide polymorphisms (SNPs) associated with canine hip dysplasia on the CFA14 chromosome and 37 in the Mountain Bernese breed with candidate genes of interest being Paraoxonase-2(PON-2) on CFA14 and Fibronectin-1(FNI-1) on CFA37. PON-2 and FNI-1 are considered to be part of the pathogenesis of canine hip dysplasia due to their involvement in bone mineral density and extracellular matrix cartilage.

Dysgen Test - Researchers from Bioiberica, University of Barcelona, and Progenica Inc. have developed a genetic test that can assess the individual probability of developing canine hip dysplasia in Labrador Retrievers. The test has an accuracy of $85 \%$, a sensitivity of $80 \%$, and a specificity of $78 \%$, but when is in conjunction with the results from the $\mathrm{x}$-ray exam those numbers increased and the test can be applied in breeding programs. The researchers tried to find the presence or absence of 7 single nucleotide polymorphisms (SNPs) which have been linked to canine hip dysplasia. At the end of the study, it was concluded that there is no significant statistical evidence to support the predictive value of the Dysgen test (Bank and Ström, 2016). Bruun et al, 2020, has conducted a study on 39 dogs from the Danish Labrador Retrievers breed. In this study, 14 dogs were with hip dysplasia (grade D or E) and 25 dogs were without hip dysplasia. The authors concluded that for their study group the Dysgen Test has no prognostic value against canine hip dysplasia.

It is considered that a genomic selection scheme will be the alternative for reducing the prevalence of canine hip dysplasia, so a genomic (DNA) test is needed as long as it can provide information about a large number of genotype markers in a population of phenotypic marked dogs. Until such a test is available, selection against canine hip dysplasia will be made according to the criteria described above (Soo et al, 2015).

\section{Treatment for canine hip dysplasia}

Treatment for canine hip dysplasia pursues two directions, conservative, drug treatments, and surgical treatments. Bell J, 2015, states in its study that about $75 \%$ of dogs diagnosed with varying degrees of dysplasia may exhibit a good quality of life and good functionality of the coxo-femoral joint only using drug treatment, without the need for surgical intervention. Nonsteroidal anti-inflammatory drugs are found in the primary medical management of CHD.

The main objectives of conservative treatment in dogs involve reducing pain, maintaining the function and range of movement of the hip, returning as quickly as possible to normal physical activity, slowing the progression of the condition, all without major adverse effects. It is also very important to maintain an optimal weight, control the pain, strengthen the periarticular muscles, maintaining healthy cartilage, and limiting inflammation. Treatment is done depending on the phase of the disease. In the acute condition, rest is necessary alongside anti-inflammatory medication, analgesic medication, and physiotherapy, while in the chronic phase it is intended to maintain a balanced diet but also physical activity is very important for a normal quality of life (Harper, 2017; Hamish, 2004).

Nonsteroidal anti-inflammatory drugs such as carprofen, ketoprofen, meloxicam, robenacoxib, tolfenamic acid, etc. are used in canine hip dysplasia, and analgesic medication such as tramadol, acetaminophen, gabapentin. To reduce weight in obese animals, protein inhibitors such as mitratapid or drilotapid may be used as a medication. Glucosamines can also be used in combination with chondroitin sulfate, omega 3 fatty acids, and hyaluronan (Harper, 2017).

Among the most common surgical interventions, is juvenile pubic simphysiodesis, triple pelvic osteotomy, total hip replacement, and femoral osteotomy (Tobias and Johnston, 2012).

- Juvenile pubic simphysiodesis: involves electrocautery of the pubic growth plate which will cause thermal necrosis of chondrocytes and premature closure of the growth plate. Is recommended to be performed around 15 weeks of age. This method is simple, fast, and does not require orthopedic implants and results in a bilateral acetabular rotation, and the return to normal activity is rapid, while complications are extremely rare (Anderson, 2011).

- Triple pelvic osteotomy: the main objective of this procedure is to prevent the subluxation of the femoral head by increasing the dorsal acetabular coverage achieved by osteotomy of the ilium, ischium, and pubis. It is recommended that the intervention be performed on patients up to 1 year of age (Anderson, 2011).

- Total hip replacement: It is a salvage procedure, which involves replacing the femoral head as well as the affected acetabular bone with implants. This intervention is carried out with caution in young dogs because there has been a higher rate of postoperative complications than in adult dogs (Vezzoni et al., 2008).

- Femoral osteotomy: It is also a salvage procedure that aims to excise the femoral head so that the pain decreases in intensity or even disappears (Tobias et al., 2012).

\section{Author Contributions:}

F.L. wrote the manuscript with support from D.L.P; R.L. and C.R.P. helped supervise the project; D.L.P. supervised the project. All authors provided critical feedback and helped shape the research, analysis, and manuscript.

\section{Acknowledgments}

This research did not receive any specific grant from funding agencies in the public, commercial, or not-for-profit sectors but the study was part of the regular monitoring of the health of SB laboratory animals.

\section{Conflicts of Interest}

The authors declare that they do not have any conflict of interest. 


\section{REFERENCES}

1. Anderson A, Treatment of hip dysplasia, Journal of Small Animal Practice, 2011, 52, 182-189

2. Bank A, Ström A, Validation of the Dysgen Hip Dysplasia DNA test in the Danish population of Labrador Retrievers, Master's thesis, 2016. Available from: https://www.dkk.dk/uploads/documents/R\%C3\%A5dgivning/Specialer/validation_of_the_dysgen_hip_dysplasia_dn a_test_in_the_danish_population_of_labrador_retrievers.pdf

3. Bell J, Clinical and Breeding Management of Canine Hip Dysplasia, World Small Animal Veterinary Association World Congress Proceedings, 2015 May 15-18; Bangkok, Thailand.

4. Bruun, CS, Bank, A, Strom A, Proschowsky, HF, Fredholm M, Validation of DNA test for hip dysplasia failed in Danish Labrador Retrievers. Animal Genetics, 2020, age.12951

5. Butler J Ryan, Gambino J, Canine Hip Dysplasia: Diagnostic Imaging, Veterinary Clinics of North America: Small Animal Practice, 2017, 47(4), 777-793.

6. Hamish RD, Management of Hip Dysplasia, 29th World Congress of the World Small Animal Veterinary Association, 2004, Oct 6-9, Rhodes, Greece

7. Harper TAM, Conservative Management of Hip Dysplasia. Veterinary Clinics of North America - Small Animal Practice, 2017, 47(4), 807-821.

8. Howe M L, Slater MR, Boothe WH, Hobson PH, Holcom LJ, Angela C, Long-term outcome of gonadectomy performed at an early age or traditional age in dogs, Spann Journal of the American Veterinary Medical Association, 2001, Vol. 218, No. 2, Pages 217-221.

9. Kealy RD, Lawler DF, Ballam JM, Lust G, Smith GK, Biery DN, Olsson SE, Five-year longitudinal study on limited food consumption and development of osteoarthritis in coxofemoral joints of dogs. J Am Vet Med Assoc. 1997 Jan $15 ; 210(2): 222-5$.

10. King MD, Etiopathogenesis of Canine Hip Dysplasia, Prevalence, and Genetics. Vet Clin North Am Small Anim Pract. 2017 Jul;47(4):753-767.

11. Krontveit RI, 2012, Canine hip dysplasia in a prospective cohort study Incidence, risk factors and long-term effects in four large breeds, Dissertation for the Degree of $\mathrm{PhD}$, Available from: http://www.longfeng.se/picts/documents/Krontveit_PhD_thesis_2012.pdf

12. Krontveit RI, Nødtvedt A, Sævik BK, Ropstad E, Trangerud C, Housing- and exercise-related risk factors associated with the development of hip dysplasia as determined by radiographic evaluation in a prospective cohort of Newfoundlands, Labrador Retrievers, Leonbergers, and Irish Wolfhounds in Norway. Am J Vet Res. 2012 Jun;73(6):838-46.

13. Kyriazis A, Prassinos NN, "Canine hip dysplasia: Part I: Aetiopathogenesis \& diagnostic approach”, Hellenic Journal of Companion Animal Medicine, 2016, 5(1), pp. 22-47

14. Merca R, Bockstahler B, Vezzoni A, Tichy A, Boano S, Vidoni B, Canine hip dysplasia screening: Comparison of early evaluation to final grading in 231 dogs with Fédération Cynologique Internationale A and B. PLoS ONE, 2020, 15(5): e0233257.

15. Mortellaro CM, Petazzoni M, Vezzoni A, ATLANTE BOA® Breed-oriented Orthopaedic Approach, Ed. Innovet, 2008.

16. Papuc I, Lăcătuș R, Radiologie și radioprotecție: Diagnosticul radiologic în afecțiunile aparatului locomotor la animale domestice, Ed. AcademicPres, Cluj-Napoca, 2013.

17. Peterson C, Canine hip dysplasia: Pathogenesis, phenotypic scoring, and genetics, The Duluth Journal of Undergraduate Biology, 2017, Vol. 4.

18. Pfahler S, Distl O, Identification of quantitative trait loci (QTL) for canine hip dysplasia and canine elbow dysplasia in Bernese mountain dogs. PLoS One. 2012;7(11):e49782

19. Saunders J, Broeckx B, Radiographic Methods for Diagnosis of Hip Dysplasia, World Small Animal Veterinary Association Congress Proceedings, 2017, Sep 25-28; Copenhagen, Denmark.

20. Schachner ER, Lopez MJ, Diagnosis, prevention, and management of canine hip dysplasia: a review. Vet Med (Auckl). 2015 May 19; 6:181-192.

21. Shipov A, Vardi E, Yudelevitc, S, Milgram J, Ultrasonographic Assessment of the Immature Canine Coxo-Femoral Joint in Accordance with Graf's Technique, Israel Journal of Veterinary Medicine, June 2019, Vol. 74 (2).

22. Soo M,Worth AJ, Canine hip dysplasia: phenotypic scoring and the role of estimated breeding value analysis, New Zealand Veterinary Journal, 2015, 63:2, 69-78.

23. Thrall DE, Textbook Of Veterinary Diagnostic Radiology, Seventh Edition, Elsevier Ltd., 2018. 
24. Tobias K, Johnston S, Veterinary Surgery Small Animal 2nd Ed., Elsevier Ltd., 2012.

25. Todhunter RJ, Mateescu R, Lust G, Burton-Wurster NI, Dykes NL, Bliss SP, et al, Quantitative trait loci for hip dysplasia in a cross-breed canine pedigree. Mamm Genome, 2005, Sep;16(9):720-30.

26. Todhunter RJ, Garrison SJ, Jordan J, Hunter L, Castelhano MG, Ash K, Meyers-Wallen V, Krotscheck U, Hayward JJ, Grenier J, Gene expression in hip soft tissues in incipient canine hip dysplasia and osteoarthritis. J Orthop Res., $2019 \mathrm{Feb} ; 37(2): 313-324$.

27. Verhoeven G, Fortrie R, Van Ryssen B, Coopman F, Worldwide screening for canine hip dysplasia: where are we now? Vet Surg., 2012 Jan;41(1):10-9.

28. Vezzoni A, Dravelli G, Vezzoni L, De Lorenzi M, Corbari A, Cirla A, Nassuato C, Tranquillo V, Comparison of conservative management and juvenile pubic symphysiodesis in the early treatment of canine hip dysplasia. Vet Comp Orthop Traumatol., 2008;21(3):267-79

29. Super Veterinary Medical Technology. Available from: www.superveterinary.com accessed on 01 May 2020 